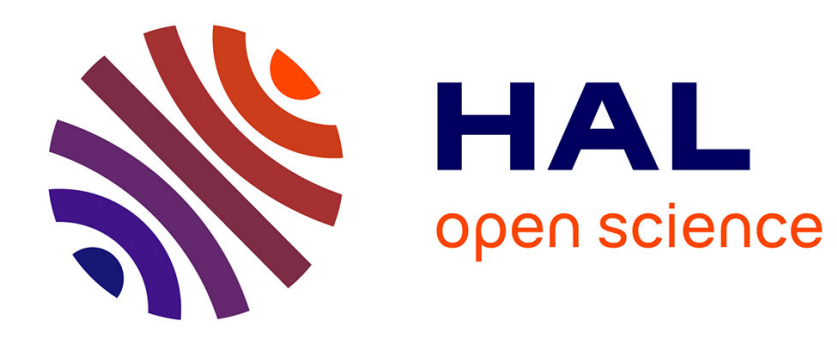

\title{
Liquid Atomization and Spray: A multi-Scale Description
}

\author{
Christophe Dumouchel
}

\section{To cite this version:}

Christophe Dumouchel. Liquid Atomization and Spray: A multi-Scale Description. ASME-FEDSM Fluids Engineering Summer Conference, Jul 2017, Waikoloa, Hawaii, United States. hal-01621280

HAL Id: hal-01621280

https://hal-normandie-univ.archives-ouvertes.fr/hal-01621280

Submitted on 12 Aug 2020

HAL is a multi-disciplinary open access archive for the deposit and dissemination of scientific research documents, whether they are published or not. The documents may come from teaching and research institutions in France or abroad, or from public or private research centers.
L'archive ouverte pluridisciplinaire HAL, est destinée au dépôt et à la diffusion de documents scientifiques de niveau recherche, publiés ou non, émanant des établissements d'enseignement et de recherche français ou étrangers, des laboratoires publics ou privés. 


\title{
FEDSM2017-69590
}

\author{
LIQUID ATOMIZATION AND SPRAY: A MULTI-SCALE DESCRIPTION \\ Christophe Dumouchel \\ Normandie Université, UNI Rouen, INSA Rouen CNRS UMR 6614 - CORIA, \\ Saint-Etienne-du-Rouvray, France
}

\begin{abstract}
A multi-scale approach to investigate liquid atomization processes is introduced. It describes the liquid system by the scale distribution whose determination is inspired from the Euclidean Distance Mapping used to measure the fractal dimension of a contour. The scale distribution is introduced in 2D and in 3D and applications from previous investigations are presented. The 2D applications are performed on experimental images and the 3D applications are performed on results obtained from Direct Numerical Simulation. The multi-scale analysis allows identifying and quantifying the mechanisms responsible for the interface evolution according to the scale. Among other results, the analyses presented here demonstrate the improvement of the atomization process when an elongation mechanism contributes to the thinning of the small structures. The multi-scale tool also provides new metrics that may be used to validate simulation results. An example of this is presented and discussed. Finally, the paper evokes several approaches to implementing the scale-distribution concept to improve or build new models.
\end{abstract}

\section{INTRODUCTION}

In many domestic or industrial applications, flows of droplets of liquids are used. It is known that the efficiency of these applications depends on the characteristics of these drops including their size, velocity, spatial density, etc. Investigations to understand, model, simulate and control liquid spray formation are therefore very much needed.

The most encountered process to produce a spray consists of ejecting a liquid flow into a gaseous environment. As soon as the liquid flow issues from the injector nozzle, deformation appear at the liquid interface. These deformations grow in space and time and eventually result in the ejection of liquid fragments on which the whole process may reiterate. The atomization process stops when the initial flow is fully transformed into a flow of droplets small enough to be stable, i.e., a spray.

The experimental investigations of atomization processes are based on visualizations and image analysis and often report measurements of characteristic features such as a spray angle, a breakup length, a mean diameter of the drops... Although these quantities are important, they don't provide a complete description of the process. Liquid atomization is a process of energy exchange between the interface and the two fluids. The liquid-gas interface stores energy, which, per unit mass, is equal to the product of the specific-surface-area (surface area per unit mass) and the surface tension [1]. The specific-surface-area is conveyed by the shape of the liquid system, the shape that continuously varies during the atomization process. The profusion of visualization approaches available in the literature [2] points out the complexity of this shape. Attempts of using the fractal concept developed to characterize the tortuosity of complex contours [3-5] have been performed to characterize this complexity [6-8]. Among other results, it was pointed out that atomizing liquid systems cannot be fully described by a single fractal dimension and that the scale range representative of the whole liquid system shape is wider than the one where selfsimilarity is observed. Thus, the traditional fractal concept must be replaced by a multi-scale description approach where the fractal dimension becomes a scale-dependent function. The notion of scale distribution has been introduced for this purpose.

The multi-scale description tool based on the measurement of the scale distribution is introduced. Coming from previous investigations, applications of this approach on experimental and simulated results are presented and discussed. They demonstrate that such a multi-scale analysis of the atomization process allows identifying the physical mechanisms responsible for the interface evolution according to the scale. Specific attention is paid on the importance of the mechanisms at small scales. This paper shows to which extend the multi-scale approach can help characterizing, understanding and modelling liquid atomization processes.

\section{THE MULTI-SCALE DESCRIPTION}

The multi-scale analysis describes the liquid system by the cumulative scale function $E_{n}(d)$ where $d$ is the scale of observation. This function is obtained by applying successive erosion operations to the liquid system. An erosion operation is 
illustrated in Fig. 1 for a 2-D system. Figure 1-left shows the initial system, which has a 2-D total surface area noted $S_{2 T}$. The system is eroded by circular structuring elements with a diameter $d$. The eroded system (dark-gray area in Fig. 1-right) has a surface area noted $S_{2}(d)$. The erosion operation is applied for $d$ varying from 0 to infinity and the cumulative surface-based scale distribution $E_{2}(d)$ is constructed as:

$$
E_{2}(d)=\frac{S_{2 T}-S_{2}(d)}{S_{2 T}}
$$

For $d=0, S_{2}(d)=S_{2 T}$. For a sufficiently large scale, the erosion operation removes all the system and the remaining surface area $S_{2}(d)=0$. Therefore in the range $d \in\left[0 ; \infty\left[, E_{2}(d)\right.\right.$ monotonously increases from 0 to 1 . The first derivative of $E_{2}(d)$ with respect to the scale is the surface-based scale distribution $e_{2}(d)$ :

$$
e_{2}(d)=\frac{\mathrm{d} E_{2}(d)}{\mathrm{d} d}=\frac{L(d)}{2 S_{2 T}}
$$

This derivative is equal to the ratio of the perimeter length $L(d)$ of the eroded system at scale $d$ on twice $S_{2 T}$.

The extension of this definition in 3-D is straightforward. In this case, the system is characterized by its total volume $V_{T}$, the erosion operation is performed with a sphere of diameter $d$ and the cumulative volume-based scale function $E_{3}(d)=\left(V_{T}-V(d)\right) / V_{T}$ involves the volume $V(d)$ of the eroded system at scale $d$. The first derivative of $E_{3}(d)$ is the volumebased scale distribution $e_{3}(d)$. This function is equal to the ratio of the 3-D surface area $S_{3}(d)$ of the eroded system divided by twice the system total volume:

$$
e_{3}(d)=\frac{\mathrm{d} E_{3}(d)}{\mathrm{d} d}=\frac{S_{3}(d)}{2 V_{T}}
$$

The distributions $e_{n}(d)(n=2,3)$ are monotonously decreasing functions, their dimension is the inverse of a length, and, they are normalized, i.e., $\int_{0}^{\infty} e_{n}(d) \mathrm{d} d=1$. Note that $e_{3}(0)$ is similar to the specific-surface-area introduced by Evers [1].

The scale distribution can be mathematically established for simple systems. For a sphere of diameter $D$, we obtain:

$$
\begin{cases}e_{n}(d)=\frac{n}{D}\left(1-\frac{d}{D}\right)^{n-1} & \text { for } d<D \\ e_{n}(d)=0 & \text { for } d>D\end{cases}
$$

For a cylinder of diameter $D$ and by considering the lateral surface only, i.e., the two circular ends are not counted, we obtain:

$$
\begin{cases}e_{n}(d)=\frac{n-1}{D}\left(1-\frac{d}{D}\right)^{n-2} & \text { for } d<D \\ e_{n}(d)=0 & \text { for } d>D\end{cases}
$$

In Eqs. (4) and (5), $n=2$ or 3 according to the dimension of the description. The diameter $D$ of these systems is equal to the maximum scale $d_{\max }$ defined as the smallest scale for which $E_{n}(d)=1$ and $e_{n}(d)=0$

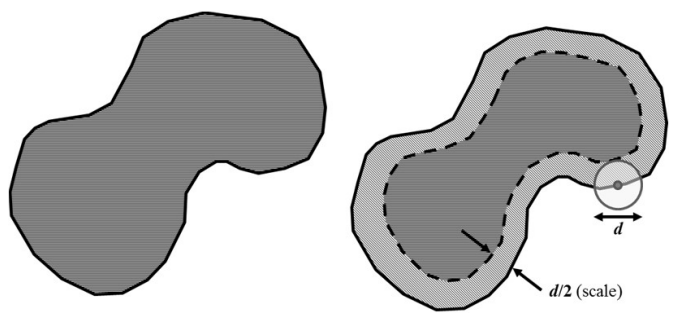

Fig. 1: Illustration of an erosion operation. Left: Initial 2-D system. Its total surface area is noted $S_{2 T}$; Right: Erosion at scale $d$. The light gray strip is removed. The eroded system has a surface area noted $S_{2}(d)$ (dark gray area)
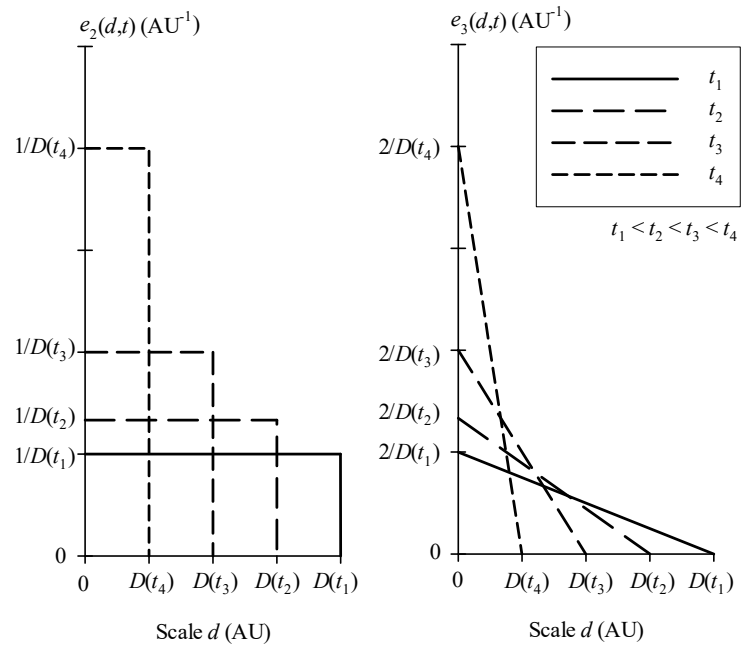

Fig.2: Temporal evolution of the scale distribution of a thinning cylindrical ligament: Left $-e_{2}(d, t)$, Right $-e_{3}(d, t)$

It is instructive to consider the case of a thinning cylindrical ligament, i.e., a cylinder with a diameter $D(t)$ that decreases with time. The temporal evolution of the scale distribution $e_{n}(d, t)$ calculated from Eq. (5) are shown in Figs. 2. The scale distributions $e_{2}(d, t)$ are successive step functions whereas the scale distributions $e_{3}(d, t)$ are successive linear functions. As $t$ increases, the characteristic features of this mechanism are a continuous increase of $e_{2}(d, t)$, a continuous decrease of the scale derivative $e_{3}{ }^{\prime}(d, t)=d e_{3}(d, t) / d d$ and a scale independence of these two functions. $e_{3}(0, t)$ indicates a constant increase of the specific-surface-area. 
Combining the quasione-dimensional continuity equation provided by Stelter et al. [9] together with the fact that the section of the cylinder is constant along the axial direction $z$, we obtain the rate of stretching $\dot{\epsilon}$, i.e.:

$$
\dot{\epsilon}=\frac{\partial v}{\partial z}=\frac{-2}{D(t)} \frac{\mathrm{d} D(t)}{\mathrm{d} t}
$$

where $v$ designates the longitudinal velocity. Considering Eqs. (4) and (5), we can express the stretching rate $\dot{\epsilon}$ of the thinning cylinder as a function of the scale distributions:

$$
\dot{\epsilon}=2 \frac{\dot{e}_{2}(d, t)}{e_{2}(d, t)}=\frac{\dot{e}_{3}{ }^{\prime}(d, t)}{e_{3}{ }^{\prime}(d, t)}
$$

where the dot indicates a temporal derivative and the prime a scale derivative. For a thinning cylindrical ligament, the stretching rate is of course independent of the scale $d$.

From a physical point of view, the thinning of a liquid ligament can be due to an elongation mechanism or a contraction mechanism. In the first case, the ligament is elongated by an external constraint and its volume is constant. In the second case, the contraction is driven by surface tension forces and expulses liquid out of the ligament whose volume therefore decreases. If for both mechanisms the specific-surface-area increases, the absolute amount of surface area actually increases during the elongation and decreases during the contraction. In the second case, the reduction of the ligament diameter is not compensated by an increase of its length. Both mechanisms are associated to a positive stretching rate and the only way to dissociate them from each other is to get an information on the temporal variation of the ligament volume.

\section{APPLICATIONS ON EXPERIMENTAL ATOMIZATION PROCESS}

This section presents two examples of the use of the multiscale approach to analyze experimental images of a liquid atomization process. The first example concerns the behavior of elongated liquid ligaments and the second one considers a turbulent liquid jet issuing from a gasoline direct injector (GDI).

\section{Elongated liquid ligaments}

The elongated liquid ligaments are those developing during the atomization of a turbulent liquid sheet. The experiment work (detailed in [10]) consisted in visualizing the behavior of the ligaments with a high-speed camera (Phantom V12, 25000 frames/s) in a shadowgraph configuration. Three liquids with a surface tension $\sigma$ ranging from 38 to $70 \mathrm{mN} / \mathrm{m}$ were used. For each liquid, several ligaments were detected and analyzed. Figure 3 shows an example of the temporal evolution of one ligament. The elongation of the ligament is recognized by the constant increase of its length during time. Being produced by a turbulent liquid sheet, the ligament is deformed at initial time.

Image analyzing tools are applied to measure the scale distribution $e_{2}(d, t)$. It first consists in determining the location of separation between the ligament and the bulk when they are still in contact. This is done by hand (see the arrow in Fig. 3). Second, the ligament contour is determined at each time and the corresponding scale distribution is measured. (Refer to [10] for details).

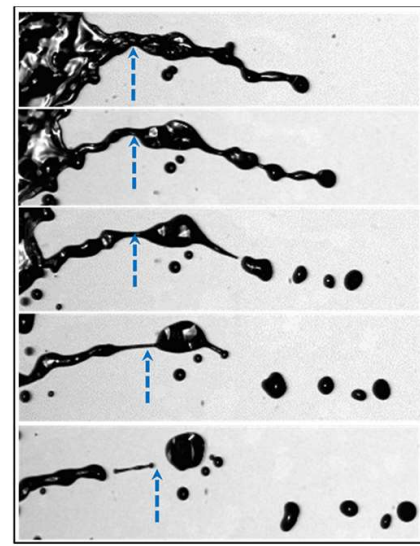

Fig. 3: Temporal evolution of an atomizing water ligament into air. Time gap between two consecutive images $80 \mu \mathrm{s}$. Spatial resolution $10.36 \mu \mathrm{m} /$ pixel. The arrow indicates the point of separation used to isolate the ligament [10]

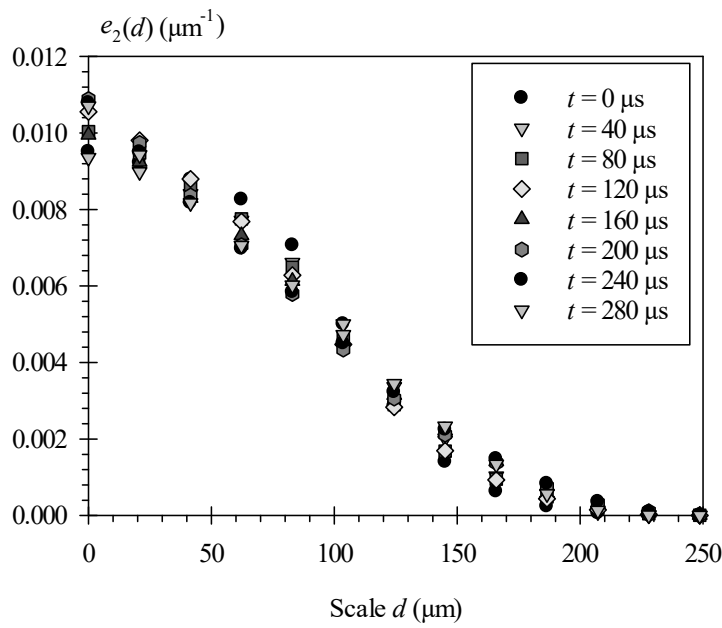

Fig. 4: Temporal evolution of the scale distribution $e_{2}(d, t)$ $(\sigma=0.0070 \mathrm{~N} / \mathrm{m})$.

Figure 4 shows an example of the temporal evolution of the scale distribution obtained for one ligament of water $(\sigma=0.0070$ $\mathrm{N} / \mathrm{m})$. As mentioned above, the distribution $e_{2}(d, t)$ is a continuous decreasing function in the scale space. At $t=0$, $e_{2}(d, t)$ resembles to a smoothed step function. The similarity with a step function reveals the global ligamentary shape of the liquid system and the smoothed feature indicates that the ligament is perturbed. (Indeed, as shown in Fig. 2-left, a non-perturbed cylindrical ligament reports a stiff step function.) At the final 
time ( $280 \mu \mathrm{s}$ in Fig. 4), the scale distribution characterizes the few drops produced after the breakup of the ligament.

Globally speaking, Fig. 4 reports an increase of $e_{2}(0, t)$ with time revealing the presence of a thinning mechanism as shown in Fig. 2-left. In the meantime, $e_{2}(d, t)$ increases with time in the large scale region $(d>130 \mu \mathrm{m})$ and $d_{\max }$ increases. This behavior demonstrates the production of bigger structures and is attributed to the presence of a thickening mechanism. Since the distribution is normalized, the combination of the thinning and thickening mechanisms imposes a decrease of $e_{2}(d, t)$ with time for the medium scale range. The thinning mechanism at small scales and the thickening mechanism at large scales are reported for each ligament. They are clearly dependent on the liquid surface tension. For instance, $e_{2}(0, t)$ increases more with time when the liquid surface tension decreases and the final scale distribution characterizing the set of droplets evolves from a linear to a convex function. Considering Eq. (4), this latter observation says that all drops produced have the same size when the surface tension is high.

The behavior of the ligaments actually results from the competition between two mechanisms: the thinning mechanism imposed by the initial ligament elongation and the thickening mechanism resulting from the development of a capillary instability. The multi-scale analysis allows quantifying this competition. Using Eq. (7), the initial elongation rate is evaluated by:

$$
\alpha\left(0, t_{i}\right)=\frac{\dot{e}_{2}\left(0, t_{i}\right)}{e_{2}\left(0, t_{i}\right)}
$$

where $t_{i}$ is the initial time. The characteristic time $t_{e}$ of the initial elongation mechanism is therefore $t_{e}=1 / \alpha\left(0, t_{i}\right)$. The capillary instability of a cylindrical ligament is a surface tension driven mechanism whose characteristic time $t_{\sigma}$ is given by (Rayleigh, 1878):

$$
t_{\sigma}=\sqrt{\frac{\rho_{L} D\left(t_{i}\right)^{3}}{\sigma}}
$$

where $\rho_{L}$ is the liquid density and $D\left(t_{i}\right)$ a characteristic length of the initial ligament. This characteristic length is the inverse of the scale distribution, i.e., $D\left(t_{i}\right)=1 / e_{2}\left(0, t_{i}\right)$. The behavior of the ligaments depends on the ratio of the capillary characteristic time on the elongation characteristic time introducing a new Weber number $W e_{i}$, i.e.:

$$
W e_{i}=\left(\frac{t_{\sigma}}{t_{e}}\right)^{2}=\frac{\rho_{L} D\left(t_{i}\right)^{3} \alpha_{i}\left(0, t_{i}\right)^{2}}{\sigma}
$$

It is found that when $W e_{i}<0.03$, the capillary instability dominates the ligament behavior and the surface tension is high enough to oppose any interface increase induced by the elongation mechanism. The resulting drops have similar size.
When $W e_{i}>0.03$, the initial elongation mechanism is strong and the surface tension doesn't oppose to the interface creation it induces. Breakup occurs at smaller and smaller liquid fragments and droplets with a wider range of diameter are produced. Therefore, an effective elongation mechanism damps the surface tension mechanism, delays the breakup process and increases the atomization efficiency. This agrees with Marmottant and Villermaux's findings [11] on the behavior of static laminar stretched ligaments.

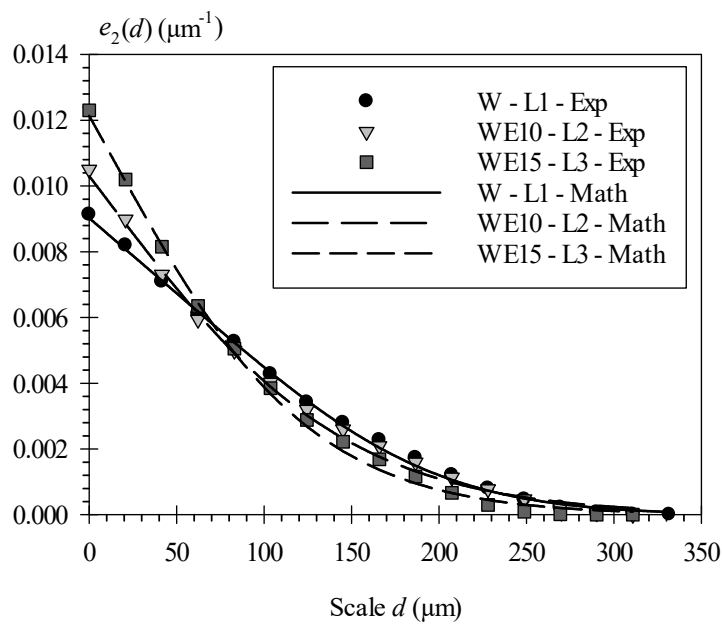

Fig. 5: Comparison between the experimental and the mathematical (Eq. (12)) scale distribution of the final droplets produced by individual ligaments (W: $\sigma=70 \mathrm{mN} / \mathrm{m}$; WE10:

$$
\sigma=44 \mathrm{mN} / \mathrm{m} \text {; WE15: } \sigma=38 \mathrm{mN} / \mathrm{m})
$$

In the same study, Marmottant and Villermaux [11] also showed that the diameter of the droplets produced by the atomization of the ligaments was distributed according to the following pdf distribution:

$$
f_{0}(D)=\frac{v^{v}}{\Gamma(v)} \frac{D^{v-1}}{D_{10}{ }^{v}} \exp \left(-v \frac{D}{D_{10}}\right)
$$

where $D_{10}$ corresponds to the mean diameter, the parameter $v$, which has no dimension, controls the width of the distribution (it decreases when the width of the distribution increases), and $\Gamma$ is the Gamma function. Since one ligament produces few droplets (see Fig. 3 for instance), the use of Eq. (11) required a statistical experimental distribution obtained from the analysis of a high number of ligaments. This is not necessary anymore if the scale distribution is used since this function is always continuous even if the system is composed of few droplets. We can demonstrate that the scale distribution of a set of spheres whose diameters are distributed according to Eq. (11) is:

$$
e_{2}(d)=\frac{2 v}{D_{10}} \frac{\Gamma\left(v+1, \frac{v d}{D_{10}}\right)-\frac{v d}{D_{10}} \Gamma\left(v, \frac{v d}{D_{10}}\right)}{\Gamma(v+2)}
$$


where $\Gamma(x, y)$ is the incomplete gamma function. The parameters $D_{10}$ and $v$ were determined for each ligament. Beside the fact that the agreements between the measurements and Eq. (12) were always good (see examples in Fig. 5), a strong correlation was obtained between the parameter $v$ and the Weber number $W e_{i}$; i.e.

$$
v \propto \frac{1}{W e_{i}}
$$

This correlation illustrates the fact that an increasing elongation mechanism ( $W e_{i}$ increases) promotes the production of smaller droplets ( $v$ decreases). The multi-scale description of the behavior of deformed and elongated ligaments demonstrated the importance of the initial elongation mechanism on the atomization efficiency and showed an interesting alternative to represent and model fragmented liquid system without statistical requirements.

\section{GDI turbulent jets}

The details of the experimental work described in this section are available in Dumouchel et al. [12]. The GDI device of this second experiments has three identical and cylindrical discharge orifices (diameter $D_{o r}=250 \mu \mathrm{m}$ ). The turbulent jet issuing from one orifice is considered only. The liquid used is Shellsol D40 whose physical properties are closed to those of gasoline. In order to ensure a visible atomization process, the injection pressure is restricted to [0.1 $\mathrm{MPa} ; 2.5 \mathrm{MPa}$ ]. The GDI device is conceived to work in transient conditions and is equipped with a needle that controls its opening and closing. The investigation concentrates on the jet behavior issuing from the injector during the fully open stage only. Contrary to the previous study, the approach is statistical, i.e., one image is taken per injection during 250 consecutive injections and the mean behavior of the jet is investigated. A shadowgraph optical arrangement with a spatial resolution equal to $5.8 \mu \mathrm{m} /$ pixel is installed. An example of the image is shown in Fig. 6-Left. The liquid jet is not laminar because of the complex internal geometry of the injector that imposes drastic flow deflections and stresses. Some of the initial perturbations grow and structure the jet evolution. This atomization process shows the production of transverse ligaments and the transformation of the bulk as a ligament network. When the injection pressure increases, more ligaments are produced. For the highest injection pressures, the bulk flow is surrounded by droplets and the jet liquid core is more indented.

Image analyzing tools are applied to measure the scale distribution. The images are orientated so that the jet appears vertical. The temporal description of the atomization process is performed on the reoriented images by analyzing a portion of the liquid system delimited by a rectangular analyzing window, and by sliding this window from the top down to the bottom of the image. The position $h$ of the analyzing window is converted into an equivalent time $t=h / V_{q}$ where $V_{q}$ is the jet mean velocity otherwise measured (Fig. 6-Right). This treatment is performed on the 250 images and a temporal evolution of the mean scale distribution is obtained for each injection pressure.
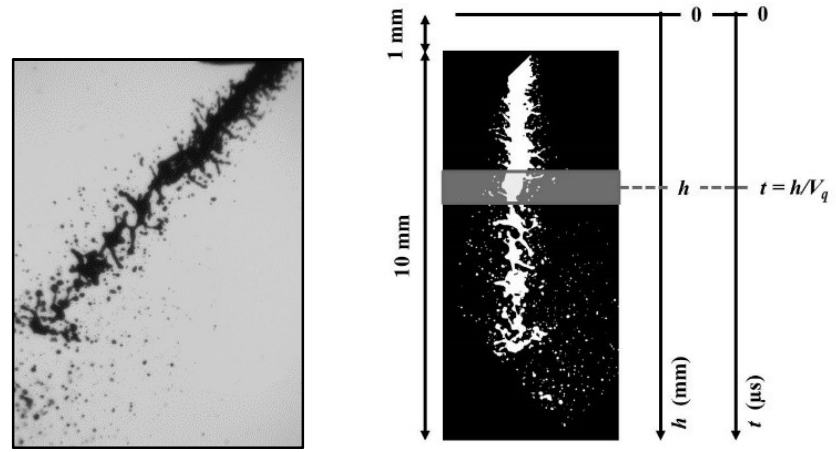

Fig. 6: Left - Image of the turbulent jet at $0.5 \mathrm{MPa}(6 \mathrm{x} 8 \mathrm{~mm})$;

Right - Local measurement of the scale distribution; The rectangle represents the analyzing window. $h$ : position of the analyzing window, Equivalent time $t=h / V_{q}$ where $V_{q}$ is the jet mean velocity

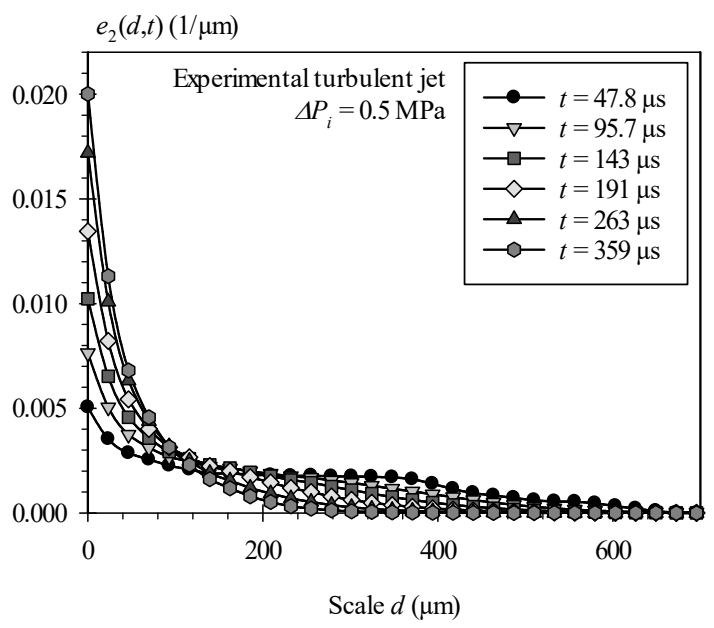

Fig. 7: Temporal evolution of the scale distribution of the turbulent jet (Injection pressure $\Delta P_{i}=0.5 \mathrm{MPa}$ )

Figure 7 shows the temporal evolution of the scale distribution obtained for $\Delta P_{i}=0.5 \mathrm{MPa}$. Similar results are found for the other injection pressures. At the smallest time, the scale distribution resembles a step function saying that the $2 \mathrm{D}$ perception of the jet is close to the one of a cylinder. The smooth decrease in the small scale range $([0 \mu \mathrm{m} ; 100 \mu \mathrm{m}])$ and in the large scale range $([400 \mu \mathrm{m} ; 600 \mu \mathrm{m}])$ result from the presence of textural (located on the interface) and structural (concerning the bulk flow) perturbations, respectively. As time goes, Fig. 7 shows an increase of $e_{2}(d, t)$ in the small scale range, a decrease of $e_{2}(d, t)$ in the large scale range and a continuous decrease of the scale $d_{\max }$. These variations are the characteristic features of a thinning mechanism. Contrary to the case of a cylindrical ligament (see Fig. 2) this thinning mechanism is scale dependent. 
The stretching rate $\alpha(d, t)=\dot{e}_{2}(d, t) / e_{2}(d, t)$ at scale $d=0$ characterizes the dynamic of the interface variation imposed by the thinning mechanism. The associate characteristic time $1 / \alpha(0, t)$ is shown as a function of time for several injection pressures in Fig. 8. This figure shows the existence of two phases in the atomization process. During the first phase, the thinning characteristic time is constant. It decreases with the injection pressure: it has been otherwise found that $1 / \alpha(0, t) \propto R^{-1.28}$ where $R e$ is the issuing Reynolds number. This reveals the dominant influence of the liquid jet turbulence on the initial stretching mechanism. During the second stage, the thinning characteristic time linearly increases with time denoting a less and less effective mechanism, i.e., a continuously decreasing specific-interface-length creation. We see that the rate of increase in this second stage is independent of the injection pressure meaning that it is not controlled by the jet dynamic anymore. The time-dependent version $T_{\sigma}(t)$ of the characteristic capillary time defined by Eq. (9) is introduced:

$$
T_{\sigma}(t)=\sqrt{\frac{\rho_{L} D(t)^{3}}{\sigma}}
$$

where $D(t)=1 / e_{2}(0, t) . T_{\sigma}(t)$ is calculated and plotted in Fig. 8 . We see that the passage from phase 1 to phase 2 always occurs when the characteristic capillary time becomes smaller that the characteristic stretching time. Therefore, during the phase 2 , the stretching mechanism is controlled by the surface tension forces explaining why its dynamic becomes independent of the injection pressure.

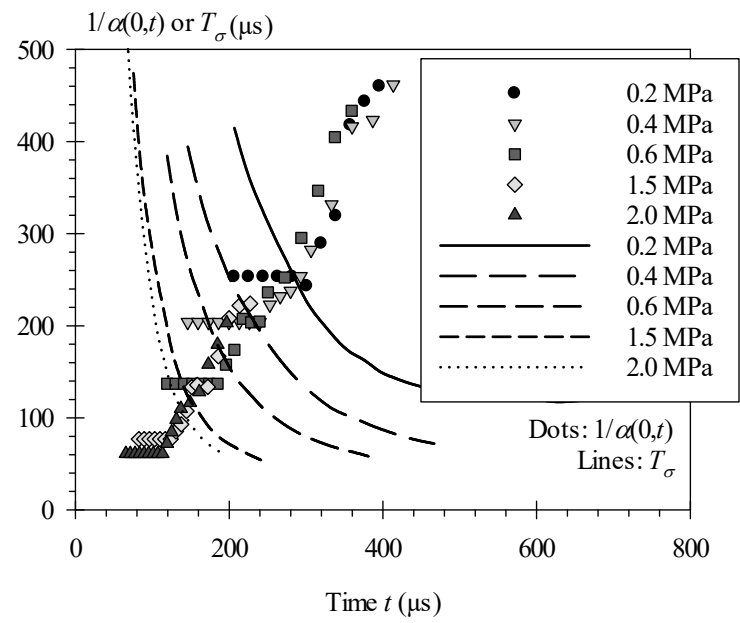

Fig. 8: Temporal evolution of the characteristic time of the thinning mechanism at $d=0$ and comparison the characteristic capillary time (several injection pressures).

In Fig. 7, the atomization process has been described as a thinning mechanism whose impact on the interface evolution follows two dynamics (Fig. 8). This thinning mechanism splits the scale space in two regions: the small scale region covering the scales for which the stretching rate is positive, and the large scale region covering the scales for which the stretching rate is negative. This space separation introduces a characteristic scale, namely, $d_{1}$, which is the smallest scale for which:

$$
\left.\dot{e}_{2}(d, t)\right|_{d=d_{1}}=0
$$

A thinning mechanism creates small scale fragments and slims large scale structures. This continuous mechanism imposes a temporal decrease of the scale $d_{1}$. This is illustrated in Fig. 9 for several pressures. The temporal decrease of $d_{1}$ is stiffer when the injection pressure increases. Furthermore $d_{1}$ decreases when the injection pressure increases. This illustrates the fact that a prompt thinning mechanism improves the atomization efficiency since it enhances the production of small liquid fragments before the breakup mechanism occurs. The beneficial influence of a rapid and strong initial thinning mechanism identified here is similar to the one observed on the elongated ligaments presented in the previous section.

Figure 9 also evidences a change of dynamic of the scale $d_{1}(t)$ (the dash lines show this change for $\Delta P_{i}=0.6 \mathrm{MPa}$ ). These dynamics correspond to the two phases of the atomization process identified in Fig. 8. Thus, the scale $d_{1}(t)$ is sensitive to the physical mechanism controlling the small scale thinning process.

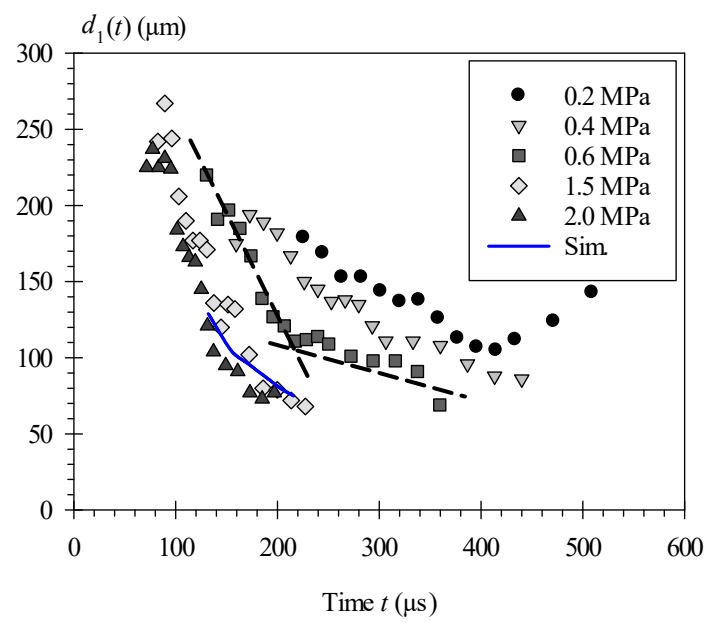

Fig. 9: Temporal evolution of the characteristic scale $d_{1}(t)$. (Several injection pressures. The dash lines visualize phase 1 and 2 of the atomization process for the $\Delta P_{i}=0.6 \mathrm{MPa}$. The blue line shows the $d_{1}(t)$ obtained from the DNS simulation)

\section{APPLICATIONS ON SIMULATED ATOMIZATION PROCESSES}

Thanks to recent developments, the direct numerical simulation (DNS) now achieves close reproduction of liquid atomization processes. Furthermore DNS allows an independent control of the initial conditions and returns a 3D information that is not reachable from experiments so far. It opens an alternative way of investigation, and, in particular, it allows performing 3D 
multi-scale analyses. Two examples are presented in this section, namely, the capillary instability of a ligament and the atomization of a turbulent jet.

Developed in the CORIA laboratory, the DNS code ARCHER used in this paper is mainly devoted to multi-phase flows. (Details on this code can be found in $[13,14]$.) The twophase flow is modelled by a single set of incompressible NavierStokes equations with variable density and viscosity, and interfacial forces. These equations are coupled with the transport equation of the phase function $C$ :

$\nabla \cdot U=0$

$\rho\left(\frac{\partial U}{\partial t}+U . \nabla U\right)=-\nabla p+\rho g+\nabla \cdot\left(\mu\left(\nabla U+\nabla U^{T}\right)\right)+F_{s t}$

$\frac{\partial C}{\partial t}+U \cdot \nabla C=0$

In ARCHER solver, the phase function is modelled with both Volume Of Fluid and Level Set methods, in the CLSVOF framework. Jump conditions across the interface are taken into account with the Ghost Fluid approach. These methods are coupled with a projection method to solve the Navier-Stokes equations on a staggered grid. To treat the convective derivative in Eq. (17) we used WENO5 scheme and 2-order central difference scheme for other. The time integration is performed with RK3TVD scheme. All details of these techniques, and how Eqs. (16-18) are solved, were presented in previous works [13, 14].

The capillary instability

The multi-scale analysis of the simulated capillary instability summarized in this section is detailed in [15]. The capillary instability manifests on a cylindrical ligament subjected to disturbances that induce surface displacements and generate a gradient of surface tension forces. Under certain conditions, the pressure distribution caused by these gradients generates internal flows that concentrate the liquid in certain regions to the detriment of others and rearrange the ligament as a succession of crests and necks. This process continues until the ligament diameter at the necks is so small that a breakup occurs and produces one drop for each swollen region. Several complete reviews on the investigations dedicated to this topic are available in the literature [16-19].

The capillary instability is calculated for a cylindrical ligament of water into air with a constant initial diameter $D_{j}=666 \mu \mathrm{m}$. An initial sinusoidal perturbation is applied. Its amplitude is fixed $\left(\eta_{0}=17 \mu \mathrm{m}, \eta_{0} / D_{j}=0.025\right)$ but eight values of the non-dimensional wavenumber $k\left(=\pi D_{j} / \lambda\right.$ where $\lambda$ is the perturbation wavelength) are considered, namely, $0.55,0.60$, $0.65,0.69,0.75,0.80,0.88,0.95$.

The simulation result for $k=0.55$ is shown in Fig. 10. The evolution of the ligament from $t=0$ to the breakup time $t_{B U}$ $(=8.13 \mathrm{~ms}$ for this case) undergoes three distinct steps. First (left column in Fig. 10), the ligament deformation increases in amplitude keeping a sinusoidal shape. Second (middle column in Fig. 10), the deformation of the ligament is not sinusoidal anymore. Two necks appear and a liquid thread develops between the main swells. Third (right column in Fig. 10), the necks do not travel anymore: they impose a local high-pressure that induces a pinch-off mechanism until breakup occurs. A similar behavior was observed for every $k$. These results are in agreement with previous calculations conducted in similar configurations [20].
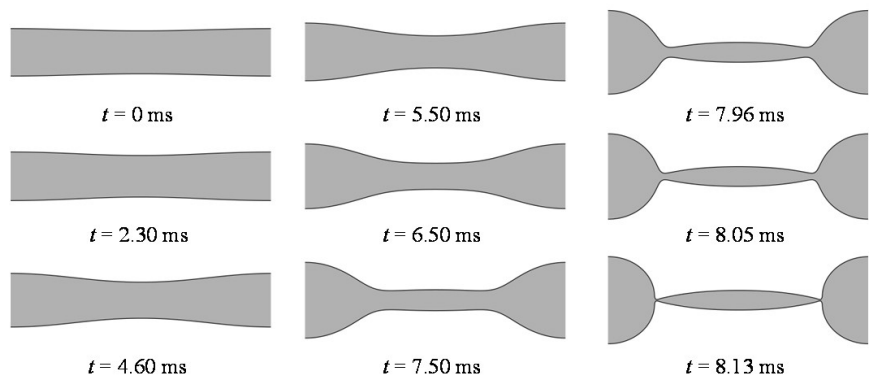

Fig. 10: DNS result: Temporal evolution of the ligament for $k=0.55$

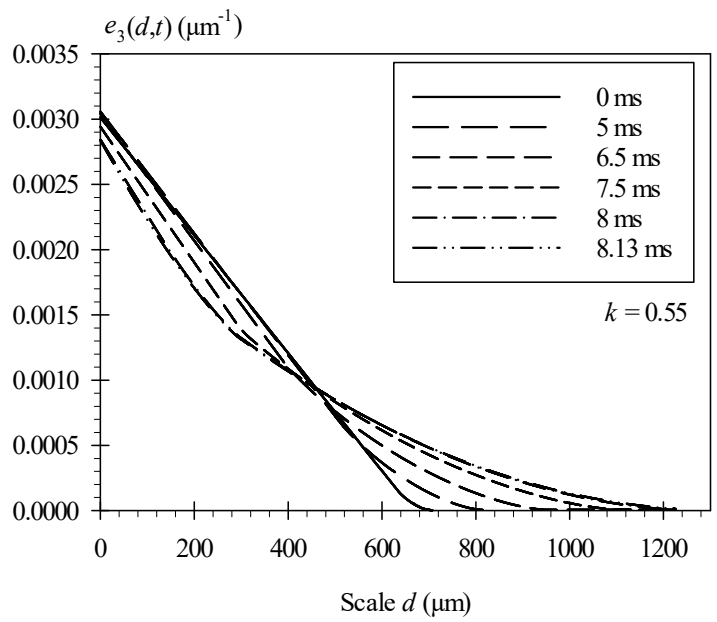

Fig. 11: Temporal evolution of the volume-based scale distribution $e_{3}(d, t)(k=0.55)$

The volume-based scale-distribution is calculated from images similar to those shown in Fig. 10 and by assuming that the ligament is axisymmetric at all times. This assumption is reasonable in the present context because axisymmetric disturbances are unstable only [21]. The temporal evolution of the scale distribution $e_{3}(d, t)$ for $k=0.55$ is shown in Fig. 11 where a thickening mechanism at large scales and a thinning mechanism at small scales are observed. The thickening mechanism is recognized by a continuous increase of the maximum scale $d_{\max }$. The thinning mechanism in the small scales is recognized by a continuous temporal decrease of the scale 
derivate $e_{3}{ }^{\prime}(d, t)$. Furthermore, since $e_{3}{ }^{\prime}(d, t)$ appears independent of the scale, this mechanism is similar to the thinning of a cylindrical ligament. The characteristic scale $d_{1}(t)$ under which the stretching rate is positive is determined as a function of time. In 3D, this scale is defined as (see Eqs. (7) and (15)):

$$
\left.\dot{e}_{3}{ }^{\prime}(d, t)\right|_{d=d_{1}}=0
$$

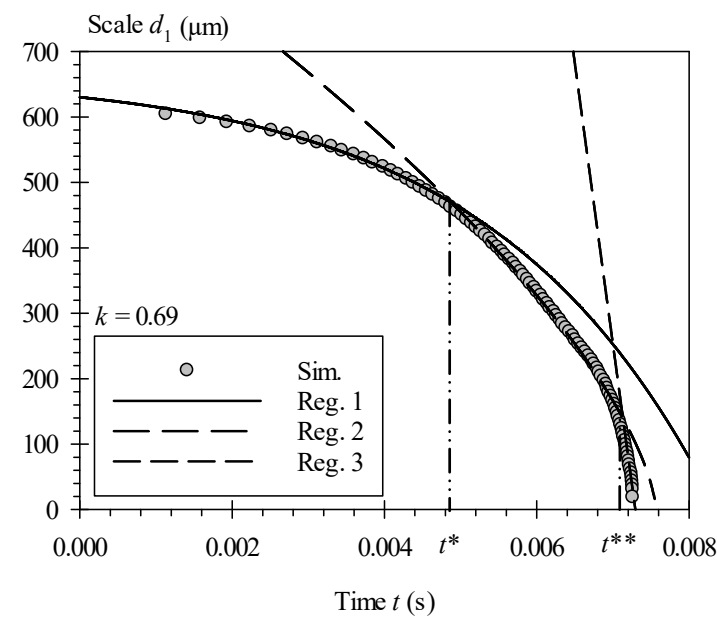

Fig. 12: Temporal evolution of the characteristic scale $d_{1}(t)$ (Lines are the mathematical representation of the three regimes)

An example of $d_{1}(t)$ is shown in Fig. $12(k=0.69)$. We see that $d_{1}(t)$ decreases according to three regimes which corresponds to the three steps identified in Fig. 10. In regime 1, $d_{1}(t)$ reports an exponential decrease with time similar to the one reported by the linear theory due to Rayleigh [21]. In regime 2, $d_{1}(t)$ decreases as $\left(\sigma t^{2} / \rho_{L}\right)^{1 / 3}$ and in regime $3, d_{1}(t)$ decreases as $\left(\sigma t / \mu_{L}\right)$. The dynamics in regimes 2 and 3 have been reported in the literature for the pinch-off mechanism at breakup [22] and the liquid-bridges contraction [23]. The existence of these three regimes demonstrates an evolution of the thinning mechanism observed at small scales.

The thinning mechanism at small scales can be characterized by the stretching rate $\dot{\epsilon}$ introduced by Eq. (7). This rate has been calculated for every wavenumber and the results are presented in Fig. 13 as a function of the ratio $t / t^{*}$ where $t^{*}$ is the time at which the regime 2 begins (see Fig. 12). Figure 13 shows a continuous increase of the stretching rate for all wavenumber. This is actually coherent with the fact that the thinning mechanism is representative of the creation of smaller and smaller liquid structures and that this phenomenon actually lasts until breakup occurs. Figure 13 also shows that the stretching rate increase when the wavenumber increases. The impact of the thinning mechanism on the specific-surface-area of the ligament can be seen in Fig. 14 that shows the temporal evolution of $e_{3}(0, t)$ as a function of $t / t^{*}$.

The specific-surface-area $e_{3}(0, t)$ first increases, reaches a maximum and then decreases to a final value smaller than the initial one. This latter point is a characteristic feature of the capillary instability which is known to reduce the total amount of surface area. The increase of the specific-surface-area corresponds to regime $1\left(t / t^{*}<1\right)$ and the maximum specificsurface-area correlates with the value of the stretching rate. The thinning mechanism during regime 1 produces interface: it is therefore an elongation mechanism. During regimes 2 and 3, the specific-surface-area decreases: the thinning mechanism becomes a contraction mechanism driven by surface tension forces. In regime 2 , this contraction mechanism is controlled by inertia; in regime 3 it is controlled by viscosity. This scenario has been confirmed by a model that is not presented here.

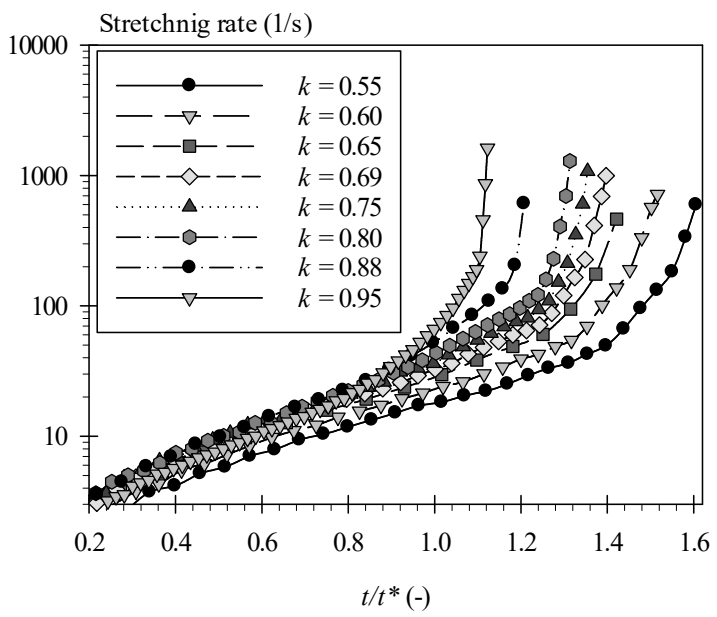

Fig. 13: Stretching rate at small scales for all wavenumbers.

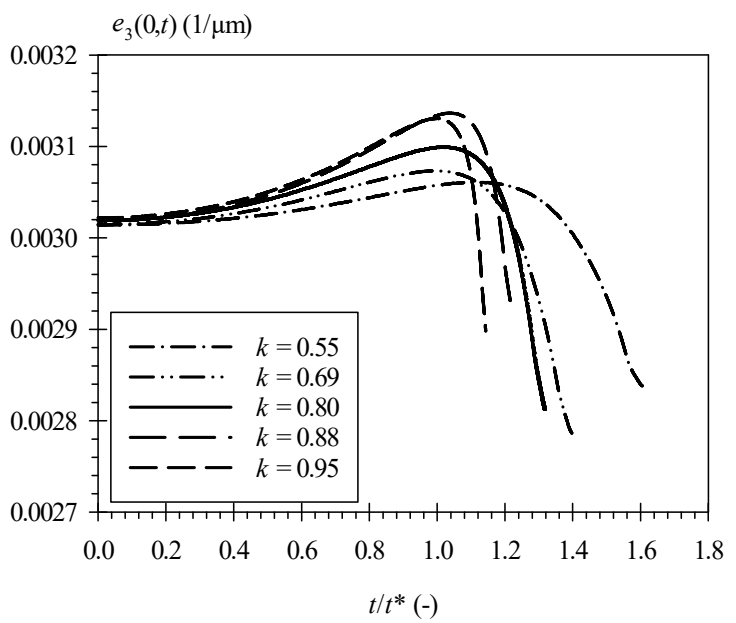

Fig. 14: Temporal evolution of the specific-surface-area (five wavenumbers)

The multi-scale analysis demonstrates the existence of an elongation mechanism at small scale even for the case of a capillary instability. Once again, it is found that this elongation mechanism is beneficial for the atomization. Indeed, we see in Fig. 14 that for $k=0.88$, the elongation mechanism is the more 
efficient since the maximum of $e_{3}(0, t)$ is the highest and the corresponding loss of specific-surface-area is the smallest. It can be added here that the characteristic times $t^{*}$ and $t_{B U}$ both increase with $k$. The elongation mechanism therefore delays the pinch-off and breakup instant. All these conclusions are valuable for the operating conditions of this study only. They might differ for other initial perturbation amplitude or wavelength since the capillary instability is known to deeply depend on these two parameters.

Simulated turbulent jet atomization process

The atomization of a turbulent jet have been calculated by the DNS code ARCHER. The liquid properties are those of the Shellsol D40. The initial diameter of the jet is $D_{j}=192.5 \mu \mathrm{m}$. The mean velocity is equal to $25 \mathrm{~m} / \mathrm{s}$. (These conditions are close to those of the turbulent jets produced by the GDI device presented above with an injection pressure equal to $\Delta P_{i}=1.1 \mathrm{MPa}$.) The initial turbulence of the jet is assumed homogenous and isotropic. It is characterize by a $5 \%$ turbulent intensity and an integral length equal to $0.08 D_{j}$. The calculation domain is $128 \times 128 \times 1024$ and the whole simulation represents an injection time of $3 \mathrm{~ms}$. Figure 15 shows an image of the simulated atomization process. At the beginning we see that the jet is perturbed by large scale disturbances only. Then, smaller and smaller disturbances appear and grow when going downstream.

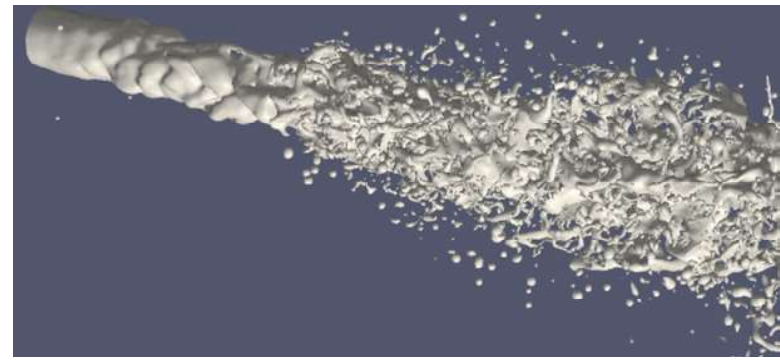

Fig. 15: Simulated atomization process of a turbulent jet. Code ARCHER

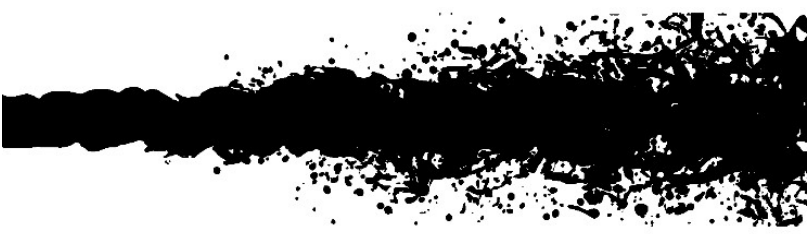

Fig. 16: 2D projection image of the $3 \mathrm{D}$ simulated jet

Two multi-scale analyses are conducted. The first one is a $2 \mathrm{D}$ approach that intends to reproduce the analysis performed in the experimental work on the GDI jets presented above. 70 images similar to the one shown in Fig. 15 are extracted from the simulation and 2D projection images of the jet are produced. (A $2 \mathrm{D}$ projection image is shown in Fig. 16.) Then, the process of $e_{2}(d, t)$ measurement developed for the experimental images (see Fig. 6) is applied on the set of 2D images. The characteristic scale $d_{1}(t)$ introduced by Eq. (15) is determined from the temporal evolution of the surface-based scale distribution $e_{2}(d, t)$. The result is reproduced by the blue line in Fig. 9. We see an excellent proximity between the experimental and simulated results from the time at which the small scale disturbances have developed. This agreement is an important result. First, it somehow validates the simulation. Second, it says that even with simplified initial conditions (which is the case here for the turbulence of the jet) the simulated atomization process may resemble the actual one after the input energy has been distributed in the scale space by the simulation. Third, this result presents a different and new procedure to validate numerical simulations.

The second analysis is $3 \mathrm{D}$. It is based on the same process as the $2 \mathrm{D}$ approach, namely, the scale distribution $e_{3}(d, t)$ is determined on a portion of the jet that is delimited by an analyzing window. This window is slid from the beginning to the end of the jet and this translation is associated with a temporal evolution of the scale-distribution. A module of 3D scale distribution measurement has been developed here and included in the ARCHER code. The $e_{3}(d, t)$ measurements have been performed on 44 positions on each image and this has been repeated on 11 images. The temporal evolution of the mean volume-based scale distribution is extracted from these measurements. This evolution is shown in Fig. 17.

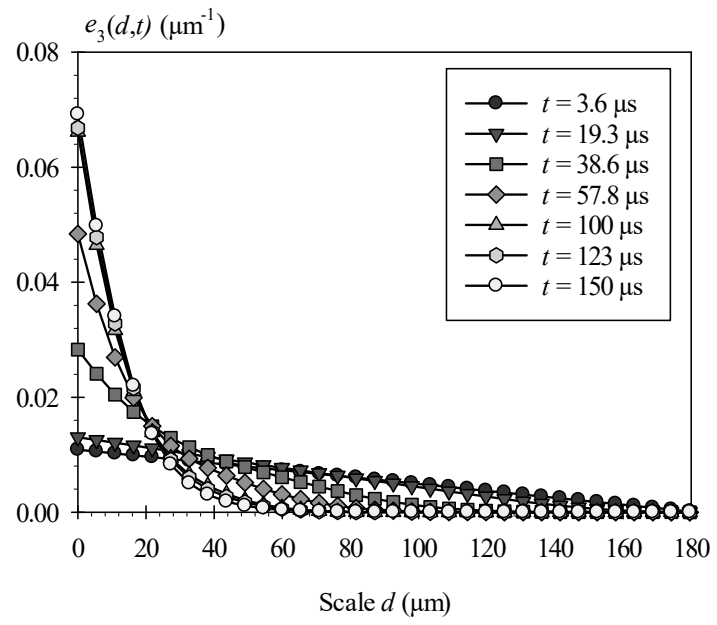

Fig 17: Temporal evolution of the volume-based scale distribution of the simulated turbulent jet.

At initial time, the scale distribution is linear indicating that the jet has an almost non-deformed cylindrical shape (see Fig. 2 ). As time goes, the simulated atomization process progresses as a thinning mechanism recognized in the small scales by a continuous increase of $e_{3}(d, t)$ and in the large scales by a continuous decrease of $d_{\max }$. (Such a mechanism was also identified on the experimental 2D scale distributions (Fig. 7).) The stretching rate introduced by Eq. (7) provides a characteristic of the thinning mechanism. In the present case, the thinning mechanism in the small scales is not similar to the one of a cylindrical ligament since the volume-based scale distribution is not linear (see Fig. 2). The stretching rate becomes 
a scale dependent function. It has been calculated for all scales and its temporal evolution is presented for some of them in Fig. 18.

At all scales, the stretching rate shows a fast increase at earlier times followed by a sharp decrease down to a value more or less close to zero. This suggests a two phase atomization process with a decrease of the stretching during the second phase. This resembles the experimental observations except for the first phase where the stretching rate is found to increase here. This of course is due to the difference in turbulent initial conditions between the experiment and the simulation. The increase of the stretching rate in phase 1 characterizes the response of the jet to the initial homogeneous and isotropic turbulence. Figure 18 shows that the effect of the turbulence is concentrated at the very beginning of the jet history and lasts a very short time. This is of course due to the fact that this turbulence is not maintained. A complementary analysis should be conducted to evaluate the degree of elongation of the thinning mechanism as a function of the characteristics of the turbulence.

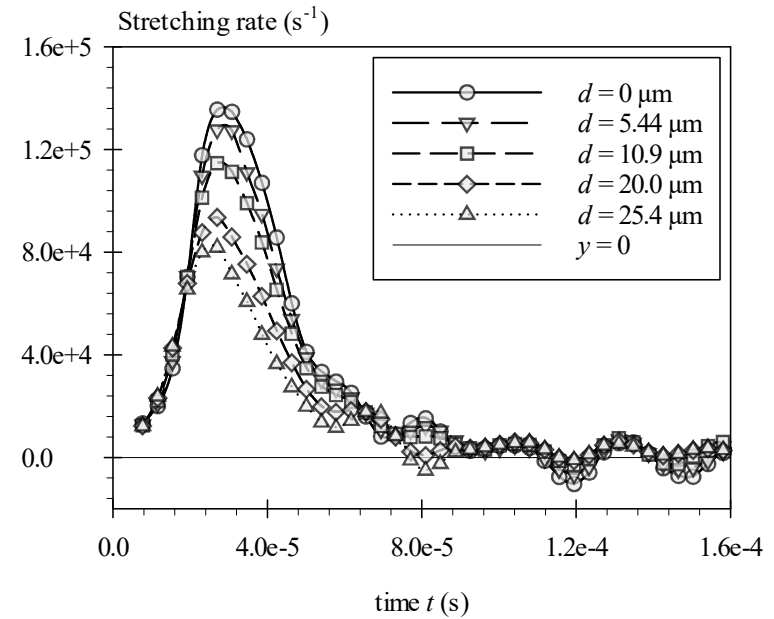

Fig. 18: Stretching rate of the 3D simulated turbulent jet as a function of the scale

Figure 18 also shows that the peak of stretching rate is higher and appears later as the scale decreases. This illustrates that the thinning mechanism operates on smaller and smaller and deformed liquid fragments only as time goes. Knowing the important influence of the thinning mechanism in small drops production, this distribution of stretching rate, which is probably connected to the decaying turbulence of the jets, might be also related to the final spray drop size distribution. This is an illustration of the new possibilities offered by the multi-scale analysis to investigate liquid atomization process and spray formation.

\section{MODELING ATOMIZATION PROCESSES}

This last short section addresses the question of the modelling of liquid atomization processes and intends to estimate the possible contribution of the multi-scale tool. Three models are rapidly introduced and discussed, namely, the model of successive fragmentations, the model of successive agglomerations and the scale-entropy diffusion model.

In the successive fragmentation model [24, 25] the atomization process is described as a succession of elementary fragmentations of spherical drops. Each mother drop breaks into daughter drops whose number-based distribution characteristics are not dependent on the diameter of the mother drop. Equating this fragmentation cascade provides the temporal evolution of the number-based diameter distribution. Using appropriate assumptions, the equation of the temporal evolution of the drop diameter distribution becomes a Fokker-Planck type equation. This model is written for high Weber number atomization process.

The model of successive fragmentations uses a diameter distribution to describe the liquid system at each time. This distribution implicitly characterizes an Equivalent System whose definition is omitted by the model. This definition must be based on a measureable characteristic of the atomization liquid system. The scale distribution could be an interesting candidate for this duty. The equivalent system could be a set of simple objects whose scale distribution is the same as the actual system. This idea is identical to the one that led to the mathematical expression for the scale distribution of the few droplets produced by the atomization of a stretched deformed ligament (Eq. (12)). This equation actually says that a set of spheres having the same scale distribution as the actual system exists. It is believed that we could extend such an approach to more complex situations.

The model of successive agglomerations is due to Villermaux et al. [26] and Villermaux [27]. It describes an atomization process as a succession of agglomerations of elementary blobs. Blobs are defined as spheres. The initial blobs diameter distribution is related to shape of the liquid system at initial time. The blobs diameter distribution evolves to the final drop diameter distribution. The temporal evolution of the blubs diameter distribution is governed by a Boltzmann-type equation. This mathematic is similar to the one encountered in the modeling of soot aggregate formation. In atomization, this model has been developed to describe the last stage of the atomization process in which liquid fragments break under the action of the surface tension forces. The drop diameter distribution reported by this model in the one given by Eq. (11). The pertinence of this distribution within the context of a multi-scale analysis has been illustrated in the investigation dedicated to the atomization of stretched deformed ligaments. It is therefore believed that the concept of elementary blob distribution used to describe the liquid system could find a concrete definition by making use of the scale distribution.

The scale entropy diffusion model has been developed by Queiros-Condé [28] to address the evolution of turbulent interfaces. It has been applied to a liquid atomization process in previous works $[29,30]$. The atomization process is described by a diffusion equation: the scale entropy diffusion equation: 


$$
\frac{\partial^{2} \Sigma(x, t)}{\partial x^{2}}-\omega(x, t)=\frac{1}{\chi} \frac{\partial \Sigma(x, t)}{\partial t}
$$

where the scale entropy $\Sigma(x, t)=\ln \left(1 / E_{2}(d, t)\right), x=\ln (d / D)(D$ being a characteristic scale of the system), $\omega(x, t)$ is the scale evolutivity function (scale-entropy flux sink function) and $\chi$ is the scale diffusivity which characterizes the ability of a perturbation to diffuse in the scale space. In turbulence, QueirosCondé [27] reported:

$$
\chi \approx\left[\frac{9}{16} R e^{3 / 2}(\ln R e)^{2}\right] /\left(l_{0}^{2} / v\right)
$$

where $l_{0}$ is the integral scale, $v$ the kinematic viscosity and $R e$ is the Reynolds. In atomization, the following expression was obtained [29]:

$$
\chi=2.9 W e_{L}^{0.555}\left(\ln \left(\frac{W e_{L}}{67}\right)\right)^{2} \frac{\sigma}{\mu^{*} D}
$$

where $W e_{L}$ is the liquid Weber number and $\mu^{*}$ is an equivalent dynamic viscosity. The similarity between Eqs. (21) and (22) is notable. The Reynolds number in turbulence (Eq. (21)) is replaced by a liquid Weber number in atomization (Eq. 22)). Although this result may appear pertinent at first glance, it questions the capacity of the model to properly take the liquid turbulence into account. A part of the answer to this question has been given in a recent investigation [12] in which it was established that the entropy diffusion model introduced by Eq. (20) is valuable in the second phase of the atomization process only, i.e., where the stretching rate at small scales are controlled by surface tension forces. In other words, a different diffusion equation should be proposed for the first phase of the atomization process during which the effect of turbulence is dominant.

\section{CONCLUSIONS}

The atomization of a liquid system in a gaseous environment is a process of continuous variation of the shape of the system until a final stable state is reached. Because of the action of the surface tension forces which characterize the liquid-gas interface, this final state is a flow of small, stable and spherical drops. Having several origins, the disturbances involved in the system deformation introduce spectra of characteristic length and time scales. Any investigation of liquid atomization processes must take this into account and therefore must be multi-scale. The present approach based on the notion of scale distribution has been motivated by this necessity. The applications described in this paper demonstrate the pertinence of the approach that improves the physical understanding of the atomization process and provides alternative tools to conduct further investigations.

An atomization process is a combination of deformation and breakup mechanisms. The multi-scale analysis shows that the deformation mechanisms may create interface whereas the breakup mechanisms consume interface. To improve the efficiency of the atomization process, the creation of interfaces during the deformation mechanisms should be favored. The multi-scale analysis allows identifying the presence of a thinning mechanism at small scales during the deformation stage. It represents the formation of small structures that develop as the breakup stage is approached and is therefore visible until this breakup event occurs. Due to the multi-scale analysis, the quantification of this mechanism is possible. It is found that the thinning mechanism is due either to an elongation mechanism or a contraction mechanism. In the first case, the interface area is increased and the breakup is delayed and operates on smaller liquid fragments. The atomization is therefore improved. Thus, mechanisms creating elongation at small scales should be favored. Those mechanisms can be imposed by the dynamic of the initial liquid system. In particular, the turbulence of the liquid system can fulfil this role. However, the present analysis reveals that the effects of the turbulence on the development of the thickening mechanism at small scales are rapid and don't last long since the turbulence is not maintained. Thus, atomization processes appear as a two-phase mechanism: during phase 1, the deformation mechanism is controlled by the liquid system dynamic, during phase 2 it is controlled by the surface tension forces.

All these results demonstrate the interesting potential of the multi-scale approach that allows handling experimental and simulation results in a more pertinent and constructive way as usually done. Furthermore, the notion of scale distribution shows an interesting potential in the domain of liquid atomization modeling.

\section{ACKNOWLEDGMENTS}

The author acknowledges the financial support from the French National Research Agency (ANR) through the program Investissements d'Avenir (ANR-10 LABX-09-01), LABEX EMC3. The author would like to thank Dr. Thibaut Ménard and Dr. Wojciech Aniszewski for the ARCHER simulations.

\section{REFERENCES}

[1] Evers, L.W. 1994 "Analogy Between Atomization and Vaporization Based on the Conservation of Energy." SAE Technical Series, Paper n ${ }^{\circ} 940190$

[2] Dumouchel, C. 2008 “On the Experimental Investigation on Primary Atomization of Liquid Streams." Experiments in Fluids 45: pp. 371-422

[3] Mandelbrot, B. 1982 “The Fractal Geometry of Nature.” WH Freeman \& Co, New-York

[4] Sreenivasan, K.R. and Meneveau, C. 1986 "The Fractal Facets of Turbulence." Journal of Fluid Mechanics 173, pp. $357-$ 386

[5] Hunt, J.C.R. and Vassilicos, J.C. 1991 "Kolmogorov's Contribution to the Physical and Geometrical Understanding of Small-Scale Turbulence and Recent Developments." Proceeding of the Royal Society London A. 434, pp. 183-210 
[6] Shavit, U. and Chigier, N. 1995 "Fractal Dimensions of Liquid Jet Interface under Break-Up." Atomization and Sprays 5, pp. 525-543

[7] Dumouchel, C., Cousin, J. and Triballier, K. 2005 "Experimental Analysis of Liquid-Gas Interface at Low-WeberNumber: Interface Length and Fractal Dimension." Experiments in Fluids 39, pp. 651-666

[8] Grout, S., Dumouchel, C., Cousin, J. and Nuglisch, H. 2007 "Fractal Analysis of Atomizing Liquid Flows." International Journal of Multiphase Flows 33, pp. 1023-1044

[9] Stelter, M., Brenn, G., Yarin, A.L., Singh, R.P. and Durst, F. 2000 "Validation and Application of a Novel Elongation Device for Polymer Solutions." Journal of Rheology 44, pp. 595-616

[10] Dumouchel, C., Blaisot, J.B., Bouche, E., Ménard, T. and $\mathrm{Vu}$, T.T. 2015 "Multi-Scale Analysis of Atomizing Liquid Ligaments." International Journal of Multiphase Flow 73, pp. 251-263

[11] Marmottant, P. and Villermaux E. 2004 "Fragmentations of Stretched Liquid Ligaments." Physics of Fluids 16, pp. 27322741

[12] Dumouchel, C., Ménard, T. and Aniszewski, W. 2015 "Towards an Interpretation of the Scale Diffusivity in Liquid Atomization Process: An Experimental Approach. Physica A 438, pp. 612-624

[13] Tanguy, S. and Berlemont, A. 2005 "Application of a Level Set Method for Simulation of Droplet Collisions." International Journal of Multiphase Flow 31, pp. 1015-1035

[14] Ménard, T., Tanguy, S. and Berlemont, A. 2007 “Coupling Level Set/VOF/Ghost Fluid Methods: Validation and Application to 3D Simulation of the Primary Break-Up of a Liquid Jet." International Journal of Multiphase Flow 33, pp. $510-524$

[15] Dumouchel, C., Aniszewski, W., Vu, T.T. and Ménard, T. 2017 "Multi-Scale Analysis of Simulated Capillary Instability." International Journal of Multiphase Flow 92, pp. 181-192

[16] Bogy, D.B. 1979 "Drop Formation in a Circular Liquid Jet." Annual Review of Fluid Mechanics 11, pp. 207-228

[17] Eggers, J. 1997 "Non-Linear Dynamics and Breakup of Free Surface Flows." Reviews of Modern Physics 69, pp. 865-929

[18] Eggers, J. and Villermaux, E. 2008 "Physics of Liquid Jets." Reports on Progress in Physics 71, 036601 pp. 1-79

[19] Ashgriz, N. and Yarin, A.L. 2011 "Handbook of Atomization and Sprays: Chapter 1-Capillary of Free Liquid Jets" (ed. N. Ashgriz,)

[20] Ashgriz, N. and, Mashayek, F. 1995 "Temporal Analysis of Capillary Jet Breakup." Journal of Fluid Mechanics 291, pp. 163-190

[21] Rayleigh, Lord, J.W.S. 1878 "On the Instability of Jets." Proceedings of the London Mathematical Society 10, pp. 4-13

[22] Lister, J.R. and Stone, H.A. 1998 "Capillary Breakup of a Viscous Threads Surrounded by Another Viscous Fluid." Physics of Fluids 10, pp. 2758-2764

[23] Clasen, C., Philipps, P.M., Palangetic, L. and Vermant, J. 2012 "Dispensing of Rheologically Complex Fluids: The Map of Misery." American Institute of Chemical Engineering Journal 58, pp. 3242-3255
[24] Gorokhovski M. and Saveliev V.L. 2003 "Analyses of Kolmogorov's Model of Breakup and its Application into Lagrangian Computation of Liquid Sprays under Air-Blast Atomization." Physics of Fluids, 15, pp. 184-192

[25] Gorokhovski M. 2003 "Fragmentation under the Scaling Symmetry and Turbulent Cascade with Intermittency." Center for Turbulent Research, Annual Research Briefs, pp. 1-7

[26] Villermaux E., Marmottant P. and Duplat J. 2004 "Ligament-Mediated Spray Formation." Physical Review Letters 92(7), 074501(4)

[27] Villermaux E. 2007 "Fragmentation." Annual Review in Fluid Mechanics 39, pp. 419-446

[28] Queiros-Condé D. 2003 “A Diffusion Equation to Describe Scale- and Time-Dependent Dimensions of Turbulent Interfaces." Proceeding of the Royal Society London A. 459, pp. 3043-3059

[29] Dumouchel C. and Grout S. 2009 "Application of the Scale Entropy Diffusion Model to Describe a Liquid Atomization Process." International Journal of Multiphase Flow 35, pp. $952-$ 962

[30] Dumouchel C. and Grout S. 2011 "On the Scale Diffusivity of a 2-D Liquid Atomization Process Analysis." Physica A, 390, pp. 1811-1825 